\title{
Les étudiantes juives de l'Est en Belgique (1918-1940). Une socialisation paradoxale
}

\section{Pascale Falek-Alhadeff}

\section{(2) OpenEdition \\ 1 Journals}

Édition électronique

URL : http://journals.openedition.org/cmc/483

DOI : $10.4000 / \mathrm{cmc} .483$

ISSN : 2684-3080

Éditeur

Fondation de la Mémoire Contemporaine

Édition imprimée

Date de publication : 1 décembre 2011

Pagination : 141-173

ISSN : 1377-1256

\section{Référence électronique}

Pascale Falek-Alhadeff, « Les étudiantes juives de l'Est en Belgique (1918-1940). Une socialisation paradoxale », Les Cahiers de la Mémoire Contemporaine [En ligne], 10 | 2011, mis en ligne le 01 décembre 2019, consulté le 14 novembre 2020. URL : http://journals.openedition.org/cmc/483 ; DOI : https://doi.org/10.4000/cmc.483 


\title{
Les étudiantes juives de l'Est en Belgique \\ (1918-1940) \\ Une socialisation paradoxale
}

\author{
Pascale Falek-Alhadeff
}

Fraîchement inscrites dans les universités belges, les étudiantes juives d'Europe de l'Est du début du $\mathrm{XX}^{\mathrm{e}}$ siècle peuvent, en théorie, prendre part à une vie sociale estudiantine riche et conviviale ${ }^{1}$. Lieu de rencontre, l'université leur permet de se confronter à la société d'accueil et de côtoyer des jeunes ayant des centres d'intérêts semblables. Mais en tant que femmes, juives, et étrangères de surcroît, quelles étaient leurs chances de prendre véritablement part à la vie estudiantine? Quels liens pouvaient-elles et voulaient-elles tisser avec leurs camarades d'étude? Quels réseaux souhaitaient-elles construire ? Ces questions touchent à la nature de l'accueil réservé aux étrangers en Belgique. Les autorités universitaires cherchaient dans la première moitié du $\mathrm{XX}^{\mathrm{e}}$ siècle à attirer ces clients étrangers, sources de rentrées financières ${ }^{2}$. En même temps, ces étudiants étaient priés de rentrer chez eux après avoir obtenu leurs diplômes. Or de nombreux Juifs de l'Est voulaient s'établir en Belgique.

Cette étude ne se limite pas aux femmes. Ne pouvant être dissociée de celle de leurs homologues féminins, la convivialité des hommes juifs étudiant en Belgique sera également prise en considération. Examiner la vie sociale des étudiants nous conduit à envisager des questions allant bien au-delà de la sphère estudiantine: ainsi l'adaptation et l'intégration des immigrés juifs en Belgique, la confrontation entre Juifs locaux et Ostjuden, les symétries potentielles entre antisémitisme et sexisme, le statut de la femme étrangère pendant l'entre-deux-

\footnotetext{
${ }^{1}$ Cet article se fonde sur les recherches menées dans le cadre de la thèse de doctorat de l'auteur, défendue en 2011 à l'Institut universitaire européen de Florence. Nous remercions tout particulièrement notre directeur de thèse, Philipp Ther, ainsi que toutes les personnes nous ayant aidée et soutenue dans cette recherche.

${ }^{2}$ Voir notamment : F. Caestecker - A. Rea - J. De Bock, Migreren voor een diploma. Studenten van buiten de Europese Unie in het Belgisch hoger onderwijs, Gand, 2009; P. Dhondt, « Foreign Students at Belgian Universities. A Statistical and Bibliographical Approach ", dans Revue belge d'Histoire contemporaine, XXXVIII, 2008, 1-2, pp. 5-44.
} 
guerres, etc. Autant de thématiques larges éclairant et nuançant notre connaissance de l'histoire des Juifs en Belgique pendant l'entre-deuxguerres.

Le cas belge est-il spécifique? Quelle forme prend la vie sociale des étudiants juifs de l'Est en Allemagne et en France ? Celles-ci ont fait l'objet d'études par Claudie Weill pour les étudiants russes en Allemagne au début du $\mathrm{XX}^{\mathrm{e}}$ siècle et par Caroline Barrera pour les étudiants étrangers à l'Université de Toulouse de la fin du XIX ${ }^{\mathrm{e}}$ au milieu du $\mathrm{XX}^{\mathrm{e}}$ siècle ${ }^{3}$. Quelques éléments de comparaison seront ébauchés afin de mieux cerner la thématique étudiée.

\section{Seules en Belgique}

Plus d'un millier de femmes juives de l'Est se sont inscrites dans les universités belges entre les deux guerres mondiales ${ }^{4}$. Elles sont originaires pour moitié de Pologne; un cinquième d'entre elles est de nationalité roumaine, la plupart étant nées en Bessarabie, région dépendant au siècle précédent de l'Empire tsariste. Ces femmes émigrent jeunes et généralement seules; elles rejoignent un ami, un frère ou un autre membre de la famille déjà installé au plat pays. La migration en chaîne explique en partie le choix de la Belgique comme pays d'accueil. La qualité et la bonne réputation des universités belges pèsent également dans leur décision. Par ailleurs, les institutions d'enseignement supérieur cherchent à recruter des étudiants « clients » étrangers. Ceux-ci constituent une manne financière indispensable pour ces établissements, qui ne peuvent se contenter des seuls étudiants belges. Les étrangers, majoritairement Européens de l'Est de

\footnotetext{
${ }^{3}$ Cl. Weill, Étudiants russes en Allemagne 1900-1914. Quand la Russie frappait aux portes de l'Europe, Paris, 1996 ; Cl. Weill, « Convivialité et sociabilité des étudiants russes en Allemagne 1900-1914 », dans Cahiers du Monde russe et soviétique, vol. 32, n 3, 1991, pp. 349-367. C. Barrera, Étudiants d'ailleurs. Histoire des étudiants étrangers, coloniaux et français de l'étranger de la Faculté de droit de Toulouse 19e-1944, Albi, 2007.

${ }^{4}$ Ces étudiantes s'inscrivent majoritairement à l'Université libre de Bruxelles, à l'Université de Liège et à l'Université de Gand. L'Institut supérieur de Commerce d'Anvers, haute école de renom, est prise en considération vu la forte présence d'étudiantes juives de l'Est s'y inscrivant. Par contre, l'Université catholique de Louvain, qui n'ouvrit ses portes aux femmes qu'en 1921 , n'était pas fréquentée par les Juifs étrangers. Nous n'y avons pas trouvé trace d'étudiantes juives et l'avons donc exclue de cette analyse. P. Falek, « A Multifaceted Image of Jewish Women at Belgian Universities during the Interwar Period », dans Journal of Jewish Identities, vol. 3/1, January 2010, Youngstown State University, pp. 25-40.
} 
confession juive, forment dans certains établissements ou facultés plus de la moitié du corps estudiantin. Les femmes étrangères ne sont pas en reste. Entre 1922 et 1940, sur les 62 femmes diplômées de l'Institut supérieur de Commerce de l'État à Anvers (ISCEA), seules 5 sont de nationalité belge ${ }^{5}$. Si l'on se fonde sur leurs prénoms, noms de famille et lieux de naissance, 50 sont d'origine juive et viennent d'Europe centrale et orientale. Il est vrai que l'ISCEA n'ouvrit ses portes aux étudiantes qu'après la Première Guerre mondiale. La surreprésentation des étrangères est néanmoins remarquable ${ }^{6}$. Ces dernières sont également nombreuses au sein de l'École de Commerce de l'Université de Liège. La formation y est courte : le diplôme s'obtient en deux ans seulement, du moins jusqu'en 1934-35. Entre 1923-34 et 1931-32, on dénombre plus d'étudiantes étrangères que de Belges inscrites à la Faculté de Médecine et à l'École de Commerce de l'Université de Liège ${ }^{7}$. On compte ainsi 53 étrangères pour 6 Belges inscrites en commerce en 1924-25, 45 pour 9 en 1927-28. Puis la tendance s'inverse lentement et on notera encore 33 pour 19 en 1931-32. Les étrangères dominent également en sciences : elles sont 12 pour 2 Belges inscrites en section Chimie à Liège en 1924-25 et 10 pour 2 en biologie.

La présence des étudiantes étrangères, en majorité juives, semble donc patente. Visibles, nombreuses, surreprésentées, quelles réactions suscitent-elles dans leurs Alma Mater ? Comment sont-elles perçues, quel accueil leur est réservé, et quelles images leur renvoie-t-on d'ellesmêmes, images correspondant ou non à leurs propres représentations ? Ces femmes souhaitent-elles et parviennent-elles à s'intégrer dans la vie estudiantine, à s'insérer dans le monde associatif, à prendre part aux débats, à devenir membres de cercles divers ? Ou bien sentent-elles la nécessité de se replier sur elles-mêmes, sur leur communauté d'origine, et ce pour quels motifs? Des tentatives de réponses seront ébauchées dans cet article, en analysant dans une première section leur

\footnotetext{
5 Annuaire de l'Institut supérieur de Commerce d'Anvers, 1852-1927, Anvers, 1928 ; Annuaire de l'Institut supérieur de Commerce de l'État d'Anvers, Notice historique de 1852 à 1952, ESB/1, Anvers, $1.2 \mathrm{n}^{\circ} 1$.

${ }^{6}$ Voir A. Grunzweig, Histoire de l'Institut supérieur de commerce de l'État à Anvers, Bruxelles, Cercle des anciens étudiants de l'ISCEA, 1977.

7 M. Capelle, «Les jeunes filles à l'Université de Liège et la carrière des diplômées, Enquête relative aux étudiantes des années 1920-1921 à 1947-1948 », dans Bulletin des Amis de l'Université de Liège, janvier-mars 1950, pp. 21-25.
} 
participation à la vie estudiantine tant juive que non juive. Dans une seconde partie, nous envisagerons leurs relations à l'Autre, les visions et stéréotypes mutuellement entretenus entre étudiants belges et Juifs de l'Est. Pour ce faire, les sources utilisées sont plurielles. Les dossiers thématiques et individuels de la Police des Étrangers conservés aux Archives générales du Royaume nous furent d'une grande utilité, de même que les archives des différentes universités. De nombreux journaux estudiantins furent dépouillés, ainsi que la presse juive. Les interviews d'étudiants nous ont également permis d'appréhender leur quotidien, leurs projets, rêves et inquiétudes ${ }^{8}$. Enfin, ces documents furent complétés, lorsque cela s'avéra possible, par des archives familiales privées transmises par les descendants de ces étudiants. Ces sources multiples permettent de donner une voix aux différents acteurs et d'envisager les questions abordées sous diverses perspectives.

\section{L'Université comme lieu d'intégration}

En théorie, les associations et cercles universitaires sont ouverts à tous, étudiants belges et étrangers. En pratique, nous le verrons, certains groupes seront enclins à refuser les Juifs, mais ils sont minoritaires. Le cumul de la judéité et du sexe féminin complexifie l'entrée des étudiantes dans ce monde associatif particulier. Les associations étudiantes sont connues pour être un univers masculin, où les femmes ne sont pas les bienvenues, notamment parce que l'alcool et le tabac y tiennent une place essentielle. Néanmoins, ce monde s'ouvre progressivement aux étudiantes, qui créent simultanément leurs propres organes représentatifs. Éliane Gubin et Valérie Piette ont démontré le rôle des associations estudiantines comme outil d'intégration des étudiantes dans l'université, contribuant à leur interaction sociale et à leur socialisation .

Dès la rentrée universitaire, de nombreux choix s'offrent aux étudiants : rejoindre un cercle facultaire, un groupe politique, un club sportif, etc. En 1928, Bruxelles universitaire, le principal organe

\footnotetext{
${ }^{8}$ Interviews conservées à l'Institut d'Études du Judaïsme (Bruxelles) et à la Fondation de la Mémoire contemporaine (Bruxelles).

9 É. Gubin - V. Piette, Emma, Louise et Marie. L'Université de Bruxelles et l'émancipation des femmes (1830-2000), Bruxelles, 2004, p. 168.
} 
estudiantin de l'ULB, recensait vingt-sept groupes étudiants, incluant les cercles de pharmacie, médecine, ingénierie, mais aussi les groupes des étudiants wallons, espérantistes, juifs, étrangers, socialistes, libéraux, et la jeunesse franc-maçonne ${ }^{10}$. Cette liste montre le dynamisme et la diversité de la vie sociale à l'ULB. Elle est agrémentée - une aubaine pour l'historien ! - de la liste des comités de ces cercles estudiantins, révélant les noms de celles et ceux qui seraient autrement restés dans l'ombre. Sur les 14 étudiantes mentionnées dans cette liste, 3 sont d'origine juive. C'est le cas notamment de Chana Moldaver, née à Rodmistrówka, en Russie, en 1904, qui fut inscrite à la Faculté de Philosophie et Lettres de l'ULB de 1922 à 1935'11. La scolarité de Chana Moldaver fut perturbée: elle s'inscrivit plusieurs fois en première candidature et ne fut pas inscrite de manière continue pendant dix années. L'historien ou la Police des Étrangers pourraient croire que Moldaver fut une «fausse étudiante », se faisant rare sur les bancs de l'université. Or sa participation à la vie estudiantine atteste du contraire. Les apparences peuvent être trompeuses. Cette femme manquait sans doute de moyens financiers et alternait années d'études et de travail. Elle étudiait une année sur deux. D'autres étudiants optaient pour suivre leurs formations en soirée ou pour ne présenter que les examens et travailler pendant l'année. Le visa étudiant, rappelons-le, permettait aux étrangers de rester en Belgique et était grandement prisé par ceux qui ne disposaient pas d'un permis de travail. L'engagement de Chana Moldaver dans le monde estudiantin se traduisit par une grande implication au sein de l'Association générale des Étudiantes (AGE) où elle fut élue trésorière en 192812. Une autre étudiante juive d'Europe de l'Est, méconnue de la postérité et pourtant bien intégrée dans le monde estudiantin belge, est Rywka Flakser. Née à Włocławek (Pologne) en 1906, elle s'inscrivit à l'ULB, fut diplômée en médecine en 1931 et en pédagogie en $1933^{13}$. Flakser s'engagea au sein du Cercle espérantiste, où elle fut nommée conseillère

${ }^{10}$ Archives de l'ULB (AULB), « Les Cercles facultaires », dans Bruxelles universitaire, numéro spécial St-Verhaegen, $10^{\circ}$ année, 1928.

11 AULB, Registre des étudiants, carte d'étudiante de Chana Moldaver.

12 AULB, « Les Cercles Facultaires. Nos Comités », dans Bruxelles universitaire, numéro spécial St-Verhaegen, 10 année, 1928.

${ }^{13}$ AULB, Registre des étudiants, carte d'étudiante de Rywka Flakser. 
en $1928^{14}$. Comme elles, des dizaines d'autres étudiantes s'impliquèrent dans le monde associatif estudiantin et plus particulièrement au sein de groupes politiques, socialistes et libéraux ${ }^{15}$.

Les étudiants étrangers aimaient se retrouver pour débattre et s'entretenir de culture et de science. Certains cercles estudiantins se montraient plus enclins que d'autres à les accueillir. On notera néanmoins que ce furent surtout les immigrés juifs de deuxième génération qui prirent part à la vie sociale estudiantine. Le Cercle d'Histoire attira nombre d'entre eux. Cette association créée en 1931 avait pour but de "favoriser la discussion de questions historiques entre condisciples et anciens condisciples $»^{16}$. Le Cercle d'Histoire élisait chaque année un comité composé d'un président, de deux viceprésidents, d'un secrétaire et d'un trésorier. L'adhésion aux principes du Libre Examen constituait une condition préalable à l'adhésion au comité. Le Libre Examen correspondait aux valeurs de Chana Zosia Perelman $^{17}$, qui prit sa carte de membre au Cercle d'Histoire en 193334 et en fut élue vice-présidente en 1936-3718. L'organisation de nombreuses conférences était une pratique courante pour cette association. Le 8 avril 1937, Chana Zosia Perelman prit la parole et intitula son intervention «Quelques aperçus sur l'histoire économique des temps modernes ». Elle fut l'une des rares étudiantes à intervenir lors de ces conférences, la majorité des communications étant le fait de professeurs. Mais l'exceptionnalité ne réside pas seulement dans son statut de simple étudiante par opposition à celui de professeur, mais bien dans le fait qu'elle est une femme, dont la prise de parole en public reste rare. Cette question fut d'ailleurs abordée au sein de l'AGE. Il fut ainsi rapporté que "moins d'occasions leur [aux étudiantes] sont offertes de défendre publiquement leurs idées; lors de conférences contradictoires, les étudiantes ne prennent presque jamais la parole,

14 AULB, « Les Cercles Facultaires. Nos Comités », dans Bruxelles universitaire, numéro spécial St-Verhaegen, $10^{\mathrm{e}}$ année, 1928, p. 43.

15 Ibid.

16 AULB, Bulletin du Cercle d'Histoire de l'ULB, 1930-1939, Liste des membres du Cercle d'Histoire de l'ULB arrêtée au ler mars 1934

${ }^{17}$ Sur Chana Zosia Perelman épouse Schneebalg, voir É. Wulliger - J.-Ph. Schreiber, « Chana Zosia dite Sophie Perelman-Schneebalg », dans J.-Ph. Schreiber, Dictionnaire biographique des Juifs de Belgique 19e-20e siècles, Bruxelles, 2002, pp. 275-276.

${ }_{18}$ AULB, Archives, E. 34.1, Liste des membres du comité du Cercle d'Histoire de 1930 à 1970 et statuts de cette association. 
parce que l'auditoire masculin chahuterait. ${ }^{19}$ Ce passage révèle la force de caractère d'une étudiante d'origine juive polonaise comme Chana Zosia Perelman, qui n'hésitait pas à prendre la parole en public.

Chana Zosia Perelman, interviewée en 1981, relate avoir eu une « double vie » lorsqu'elle était étudiante, participant aux activités des étudiants juifs et non juifs : « Mon frère [Chaïm] et moi [...], nous nous sentions proches de ces étudiants étrangers. Au fond, je menais une double vie. J'étais, d'une part, dans le Cercle d'Histoire, où j'étais viceprésidente et où je menais une vie d'étudiante, de « poil », insouciante belge - naturellement, je n'aimais pas boire! Et je me souviens de ce jour où tous les étudiants wallons chantaient leurs chansons wallonnes, les étudiants flamands chantaient le Vlaamse Leeuw et moi j'étais seule à chanter la Brabançonne. D'autre part, j'étais très active chez les étudiants juifs, où j'étais vice-présidente, secrétaire. Nous avions intérêt, un grand intérêt à suivre ces étudiants qui venaient de Palestine, de Pologne, de Hongrie, de divers pays, pour échapper, pour pouvoir faire des études librement. C'étaient des gens de valeur, que nous trouvions beaucoup plus intéressants que les étudiants belges, qui ne songeaient qu'à boire et à préparer leur "bloque" [préparer leur session d'examen], et qui ne voyaient pas d'autres grands intérêts dans la vie ! 20 $^{20}$

Chana Zosia Perelman ne fut pas la seule étudiante déchirée entre ses origines et son pays d'adoption. D'autres étudiants juifs, immigrés de première et de seconde génération, s'impliquèrent comme elle dans plusieurs groupes, dont le Cercle d'Histoire, un espace d'ouverture particulier. En 1933-34, David Katzenellebogen et Marc Schreiber rejoignirent le Cercle. Un an après, trois autres étudiants juifs firent de même : Armand Grunzweiger, qui publiera en 1977 une monographie sur l'Institut supérieur de Commerce de l'État à Anvers (ISCEA) ${ }^{22}$, Félix Oppenheim et Fela Liwer, qui acheva ses études d'histoire la même année et devint en 1935 la belle-sœur de Chana Zosia Perelman. En 1935-36, ce fut au tour d'Elsa Gottschalck de joindre l'association.

19 AULB, « Rubrique des Cercles. A.G. des Étudiantes », dans Bruxelles universitaire, 9 janvier 1935.

${ }^{20}$ Institut d'Études du Judaïsme (IEJ), interview de Sophie Perelman-Schneebalg par Daniel Dratwa, Bruxelles, 1981, pp. 17-18.

${ }^{21}$ D. Dratwa et J. Ph. Schreiber, «Armand Grunzweig », dans J.-Ph. Schreiber, op. cit., p. 144.

${ }^{22}$ A. Grunzweig, op. cit. 
Elsa Gottschalck habitait Anvers et se rendait quotidiennement à Bruxelles pour y suivre ses cours; l'année suivante, Moïse Szeftel, chargé de cours à l'ULB, Claudine Libovitz, étudiante en archéologie et Jacques Likvermann, prirent leurs cartes de membres ${ }^{23}$. En 1936-37, le Cercle d'Histoire comptait 108 membres, dont près de $10 \%$ étaient d'origine juive, pour la plupart appartenant à la seconde génération de Juifs immigrés en Belgique.

Quelques étudiantes plus connues furent également à l'œuvre dans ce rapprochement entre étudiants juifs et non juifs. Il faut noter l'engagement de Germaine Alechinsky au sein de la Fédération bruxelloise des Femmes universitaires, dont elle fut élue secrétaire en 192924. Germaine est la tante du peintre de renom Pierre Alechinsky.

Une autre figure exemplaire est celle des sœurs Burstenbinder. Régine naquit à Anvers en 1910, elle étudia le droit de 1928 à 1933 à l'ULB et y fit la rencontre de son époux, Pierre Van Hentenryk, étudiant belge en chimie très impliqué dans le milieu estudiantin ${ }^{25}$. Régine Burstenbinder adora ses années d'universités. Elle prit plaisir à étudier, à s'investir dans la vie sociale et culturelle. Elle avait l'habitude d'aller danser aux bals ${ }^{26}$. Dynamique et investie dans ce qu'elle entreprenait, elle fut nommée vice-présidente du Cercle de Droit en 1930 et vice-présidente des Étudiants libéraux en $1933^{27}$. L'Université était pour elle un lieu d'engagement mais aussi de rencontres. Elle en gardera de nombreuses et solides amitiés. Sa sœur, Lily Burstenbinder, de deux ans sa cadette, entama également des études de droit $^{28}$. Engagée elle aussi dans le monde associatif estudiantin, elle devint secrétaire de l'AGE en 1931-32 puis présidente de cette association un an après ${ }^{29}$. Les sœurs Burstenbinder appartenaient à la seconde génération d'immigrés juifs de l'Est en Belgique. Leurs parents étaient originaires de Cracovie. Leur père, courtier en diamants ayant une excellente situation à Anvers, les

${ }^{23}$ AULB, Archives, E. 34.1, liste des membres du comité du Cercle d'Histoire de 1930 à 1970 et statuts de cette association.

${ }^{24}$ É. Gubin et V. Piette, op. cit., p. 146.

${ }^{25}$ AULB, Registre des étudiants, carte d'étudiante de Régine Burstenbinder.

${ }^{26}$ Interview par l'auteur de Denise Van Hentenryk, l'une des filles de Régine Burstenbinder-Van

Hentenryk, Bruxelles, 5 janvier 2011.

${ }_{27}$ É. Gubin et V. Piette, op. cit., p. 180

${ }^{28}$ AULB, Registre des étudiants, carte d'étudiante de Lily Burstenbinder.

${ }^{29}$ AULB, Archives Ancien Secrétariat, 53.22 Cercles estudiantins. 
encouragea à poursuivre des études supérieures afin d'obtenir un emploi stable et rémunérateur. Il finança leurs loyers à Bruxelles ainsi que, très probablement, leurs loisirs ${ }^{30}$. L'activité sociale estudiantine des sœurs Burstenbinder les mena à figurer sur la liste des nominées pour l'élection de la « Reine des Plumes » parue en janvier 1933 dans Bruxelles universitaire ${ }^{31}$. Après vote des étudiants, parmi les douze meilleurs scores, Lily Burstenbinder obtint la cinquième place avec 399 voix et sa sœur Régine la dernière avec 49 voix ${ }^{32}$. Cependant, elles ne s'engagèrent pas dans les associations étudiantes juives, où elles se sentaient sans doute exclues - nous y reviendrons.

\section{Les cercles estudiantins}

Si être élue « Reine des Plumes » était un honneur et attestait de la popularité d'une étudiante, des élections étaient également organisées pour désigner le «Prince des Manchabals », dénigrant l'étudiant le plus assidu, studieux, courtisant les professeurs, et donc le plus impopulaire. Parmi les vingt étudiants présélectionnés par Bruxelles universitaire figurait Chaïm Perelman, le frère aîné de Chana Zosia Perelman $^{33}$. Le journal estudiantin le décrivait comme suit : «Philosophe et emmerdeur-né. Répond au prénom de Chaïm, mais cela il n'en peut rien. Emmerde jusqu'aux professeurs eux-mêmes qu'il pourchasse sans cesse à la recherche d'un "bedi renseignement". Allie le goût de la philosophie pure aux intérêts mercantiles les plus bas. Explique - sans bien entendu le justifier - l'antisémitisme. " ${ }^{34}$ Chaque candidat fut caricaturé de la sorte par la rédaction. Qualifié pour le second tour de scrutin, Chaïm Perelman fut élu «Prince des Manchabals » avec 237 voix $^{35}$. Perelman était un excellent étudiant, plus jeune que ses camarades, vu qu'il était entré à l'université à l'âge de 16 ans. Il mena une carrière exceptionnelle. Il est considéré comme

\footnotetext{
${ }^{30}$ Interview par l'auteur de Denise Van Hentenryk, l'une des filles de Régine Burstenbinder-Van Hentenryk, Bruxelles, 5 janvier 2011.

${ }^{31}$ AULB, « Élection de la Reine des Plumes », dans Bruxelles universitaire, décembre 1932, p. 12.

32 AULB, «Élection de la Reine des Plumes. Sprint final. Résultats décisifs », dans Bruxelles universitaire, février 1933, p. 5.

33 J.-Ph. Schreiber, « Chaïm Perelman », dans J.-Ph. Schreiber, op. cit., pp. 271-274.

${ }^{34}$ AULB, « Élection du Prince des Manchabals », dans Bruxelles universitaire, 25 janvier 1934.

35 AULB, «Élection du Prince des Manchabals. Deuxième tour de scrutin », dans Bruxelles universitaire, mai 1934.
} 
le fondateur de la «Nouvelle Rhétorique» et joua un rôle prépondérant dans la Résistance pendant la Seconde Guerre mondiale.

L'engagement dans des cercles facultaires constituait une pratique masculine, comme l'atteste la faible participation des étudiantes juives à ces groupes estudiantins. En 1930, Paul M.-G. Lévy fut nommé président du Cercle Solvay, tandis qu'un certain Joukovski, étudiant en chimie, fut le porte-drapeau de l'Association générale des Étudiants et Claude Weill le vice-président du Cercle des Sciences ${ }^{36}$. Marc Askenazi fut nommé questeur du Cercle des Sciences et, en 1934, il fut élu à la tête du Cercle de Médecine ${ }^{37}$. Askenazi était décrit comme un étudiant enjoué, défendant corps et âme ses idéaux politiques et surtout à l'œuvre dans des « beuveries quantitatives grandioses $»^{38}$. Aimant tous les plaisirs sauf le tabac, il était admiré de ses camarades qui décidèrent unanimement de le nommer aux plus hautes fonctions de leur cercle. Cette description des étudiants dans leur organe de presse révèle l'importance de l'atmosphère masculine qui dominait dans ces cercles et le rôle essentiel joué par la consommation de boissons alcoolisées. À ce sujet, Paul Lévy semblait avoir totalement adopté les coutumes estudiantines belges. Président du Cercle Solvay, il organisa la visite d'une brasserie et encouragea ses camarades à développer leurs « instincts bachiques plutôt que commerciaux » ${ }^{39}$. Les membres du Cercle Solvay se seraient mis à chanter, à émettre des bruits déplaisants, à créer du désordre et seraient rentrés à l'Université complètement saouls, ce qui ne fit guère plaisir à leur professeur, dont l'heure de cours fut perdue. Cette histoire se conclut pour Paul Lévy par la perte de son poste de président et par la fin de la reconnaissance officielle du Cercle Solvay comme cercle étudiant par les autorités de l'ULB... Paul Lévy figure parmi les quelques «poils » dont la biographie et le portrait furent publiés dans le journal Bruxelles universitaire, ce qui s'explique plus que probablement par sa présence au sein du comité éditorial de cette feuille de chou ${ }^{40}$.

${ }^{36}$ AULB, « La Vie universitaire », dans Bruxelles universitaire, octobre 1930, p. 10.

${ }^{37}$ AULB, « La Vie universitaire », dans Bruxelles universitaire, St-Verhaegen 1930, p. 16.

${ }^{38}$ AULB, « Marco Ashkenasi. Président du C. M. », dans Bruxelles universitaire, novembre 1934.

${ }^{39}$ AULB, Le vieux zèbre «Cercle Solvay, Lévy et Cie », dans Bruxelles universitaire, décembre 1930, pp. 16-17.

${ }^{40}$ AULB, Paul Lévy « Lévy », dans Bruxelles universitaire, St-Verhaegen 1931, pp. 20-21. 
Paul Lévy est décrit par ses camarades d'études comme aimant boire avec ses pairs, étudiant lorsque cela s'avérait impérativement nécessaire et préparant toujours assidûment ses conférences et rapports pour l'Institut Solvay, pour les semaines sociales ${ }^{41}$ de l'Institut de Sociologie et pour la synagogue ${ }^{42}$. Ses origines juives sont soulignées tant dans le texte que dans la caricature [Illustration 1] publiée dans Bruxelles universitaire par la forme et taille de son nez. Deux mois après cette publication, un autre article mentionnait que, grâce à la popularité acquise par Lévy dans ledit journal, notamment par cette inoubliable caricature, il fut nommé chargé de cours à l'École des Hautes Études à Gand ${ }^{43}$.

\section{Le sport comme facteur d'intégration}

Le sport constituait un facteur d'intégration pour les étudiants juifs belges et étrangers, phénomène intéressant car à l'opposé du processus d'exclusion existant en Allemagne ${ }^{44}$. En Belgique, les tournois universitaires rassemblaient de nombreuses équipes dans des disciplines variées. L'examen des listes des étudiants y participant permet de noter le nombre élevé d'hommes juifs d'Europe centrale et orientale, et ceci particulièrement à l'Université de Gand ${ }^{45}$. La plupart d'entre eux étaient élèves ingénieurs ou médecins. Ils excellaient surtout au football. C'était le cas d'Israël Kroll et d'Azriel Cornfeld. D'autres, comme Meer Tomachpolsky, Joseph Goldberg, Victor Mandelstam et Salomon Burdo, se révélaient gymnastes d'exception ${ }^{46}$. Former des équipes sportives constituait l'un des trois objectifs

\footnotetext{
${ }^{41}$ Sur les semaines sociales organisées à l'Institut de Sociologie voir : V. Piette, Origines, historique et contenu des semaines sociales de l'Institut de Sociologie (1912-1950). Du productivisme d'Ernes Solvay à la formation intellectuelle d'une élite, mémoire de licence, département d'Histoire, ULB, Bruxelles, 1992-1993; Ead., « Les Semaines sociales (universitaires) de l'Institut de Sociologie », dans G. Kurgan-Van Hentenryk (éd.), Laboratoires et réseaux de diffusion des idées en Belgique (XIX ${ }^{e}-X X^{e}$ siècles), Bruxelles, 1994, pp. 77-94.

42 AULB, Le vieux zèbre « Cercle Solvay, Lévy et Cie », dans Bruxelles universitaire, décembre 1930, pp. 16-17.

43 AULB, "Échos et Potins. Les Rocambolesques et très véridiques aventures de Lévy ou la publicité de B.U. paie ! », dans Bruxelles universitaire, janvier 1931, p. 18.

${ }^{44}$ Cl. Weill, op. cit., pp. 155-183.

45 AULB, « Championnats universitaires. Numéro spécial sports », dans Bruxelles universitaire, mars 1926 .

${ }^{46}$ Ibid.
} 
principaux de l'Association des Étudiants juifs de Gand en 192247. Ces amateurs de sport s'étaient sans doute déjà entraînés dans leur pays d'origine et pouvaient poursuivre leur hobby en Belgique, au sein de l'un des clubs sportifs juifs qui se développèrent durant l'entre-deuxguerres. Ce faisant, ils se mêlèrent notamment au milieu ouvrier, rassemblé sous la bannière du Club sportif des Ouvriers juifs à Anvers, le Yask. Ces clubs organisaient des tournois et la visite de clubs d'autres pays était monnaie courante ${ }^{48}$. Une affiche [Illustration 2] traduit bien ce dynamisme sportif, créateur de liens sociaux et facteur d'intégration. Elle annonce notamment un match de football en $3^{\mathrm{e}}$ division entre le Maccabi d'Anvers et La Gantoise. Le terrain de jeu devenant un espace neutre, socialisant et convivial.

Si le sport rassemblait et symbolisait la mixité sociale, quelques associations étudiantes furent critiquées pour leur manque de multiculturalisme et d'ouverture. Les groupes politiques s'incriminaient mutuellement et tentaient de prouver leur largeur d'esprit en soulignant notamment la présence d'étudiants étrangers dans leurs comités. Un article publié dans L'Universitaire et Socialistes unifiés le 15 janvier 1939 entend bien le faire savoir ${ }^{49}$. L'auteur, sous le pseudonyme d'Evo Dyl, se proclame «judéo-marxiste » et avance fièrement que le comité éditorial de ce journal estudiantin n'est pas composé uniquement d'étudiants belges mais aussi d'étudiants d'origines variées. Sur les sept principaux contributeurs de L'Universitaire et Socialistes unifiés, on compte trois étudiants d'origine juive (Josianne Bloch, Fernand Lecocq et Evo Dyl) et deux femmes. Surtout, tous sont communistes. Les associations politiques parvinrent à attirer de nombreux étudiants et étudiantes juives. Nous devons nous référer aux travaux de Rudi Van Doorslaer, José Gotovitch et Jean-Philippe Schreiber pour tenter d'avoir une vue plus complète sur la question. Parmi ces étudiantes politisées actives dans des groupes non juifs se trouvait notamment Jeanne Goldsobel. Née à Bruxelles en 1914, d'origine polonaise, elle étudiait le droit à l'ULB. Au cours de ses études, militante active, elle fut nommée à la tête des

${ }^{47}$ AUG (Archives de l'Université de Gand), 4A 24107 1922-1923, 323b, Studentenverenigingen.

48 YIVO Archives, New York, Trocki Collection, 30, Sport Clubs.

49 E. Dyl, « Humour, humour quand tu nous tiens!», dans L'Universitaire et Socialistes unifiés, 15 janvier 1939. 
Étudiants socialistes unifiés de l'ULB, fait d'autant plus exceptionnel qu'elle était une femme. Jeanne Goldsobel joua un rôle actif dans la Résistance pendant la Seconde Guerre mondiale. Arrêtée, elle fut déportée à Ravensbrück en août 1942 et assassinée à Auschwitz en octobre de la même année ${ }^{50}$. Les étudiantes politisées se retrouvent dans toutes les mouvances. Elles prennent part activement aux manifestations, distributions de tracts et débats politiques, au risque de se faire expulser du pays vu leur statut d'étrangères. On citera notamment Hava Groisman, mieux connue sous le nom d'Yvonne Jospa $^{51}$, Nadya Reznic ${ }^{52}$ et Buntea Crupnic ${ }^{53}$, actives au Parti communiste, et Shifra Werber, convaincue par le Linke Poale Zion ${ }^{54}$.

Pour conclure, des dizaines d'étudiants juifs s'engagèrent au sein d'associations non juives, espaces d'interaction par le biais d'activités culturelles, ludiques et politiques. La vie sociale estudiantine contribua grandement à la convivialité des étudiants, belges et étrangers. Néanmoins, il faut préciser qu'à l'ULB notamment, ce sont surtout les étudiantes juives immigrées de deuxième génération qui prennent part activement à cette vie sociale particulière, les étudiantes migrantes disposant généralement de moins de temps et d'argent. Mais d'autres

50 J.-Ph. Schreiber, « Goldsobel Jeanne Roxane, dite Jeanine », J.-Ph. Schreiber, op. cit., p. 133 M. Steinberg, «Quelques figures de femmes juives réfugiées en Belgique dans l'entre-deuxguerres », intervention à la conférence Exhumer l'histoire des femmes exilées politiques, ULB Bruxelles, 12 mai 2008, inédit.

51 A. Morelli - J.-Ph. Schreiber, « Sources et méthodes pour l'histoire des migrantes militantes. Le cas d'Yvonne Jospa », dans Sextant, n 21-22, 2004, pp. 277-293 ; C. Marissal, « Hava GroismanYvonne Jospa », dans É. Gubin - M.-S. Dupont-Bouchat - J.-P. Nandrin, Dictionnaire des femmes belges : $19^{e}$ et $20^{e}$ siècles, Bruxelles, 2006, pp. 293-295; S. Belli, « Des assistantes sociales en Résistance. Note sur Yvonne Jospa et Ida Sterno », dans Les Cahiers de la Mémoire contemporaine, $\mathrm{n}^{\circ}$ 7, 2006-2007, pp. 37-55 ; Fondation de la Mémoire Contemporaine (FMC) interview d'Yvonne Groisman-Jospa par Martine Goldberg, Bruxelles, 1995-1996, 2 vol.

${ }^{52}$ Sur Nadya Reznic, voir R. Van Doorslaer, Enfants du Ghetto. Juifs révolutionnaires en Belgique (1925-1940), Bruxelles, 1997, p. 58 ; J. Gotovitch, Du Rouge au Tricolore. Les Communistes belges de 1939 à 1944. Un aspect de l'histoire de la Résistance en Belgique, Bruxelles, 1992, p. 561.

${ }^{53}$ Sur Buntea Crupnic, voir J. Gotovitch, « Partisanes et militantes : femmes communistes dans la Résistance en Belgique », dans R. Vandenbussche (éd.), Femmes et résistance en Belgique et en zone interdite (1940-1944), Lille, 2006, pp. 73-86; M. Steinberg, « Quelques figures de femmes juives réfugiées en Belgique dans l'entre-deux-guerres ».

${ }_{54}$ Discussions avec Moshe Werber, fils de Shifra et d'Abusz Werber, $1^{\text {er }}$ et 5 février 2010 ; FMC, Interview de Shifra Werber par Jean-Philippe Schreiber, Tel Aviv, 1989, cassette I; Abusz Werber, Tsu der ershter Yortzayt, Tel Aviv, 1976; Abusz Werber, Fun unter der Pen. Publitsistik, redes, briv tsu yidishe schreibers. Vegn Abush Verber liser tsu zein ondenk, Tel Aviv, 1986; J.-Ph. Schreiber, « Abusz Werber », dans J.-Ph. Schreiber, op. cit., 2002, p. 357. 
raisons que strictement financières poussent les étudiants juifs d'Europe centrale et orientale, hommes et femmes confondus, à rester entre eux, repliés sur eux-mêmes. Ces causes de repli méritent qu'on s'y attarde.

\section{L'université comme lieu de repli}

La proportion des étudiants étrangers varie d'une université à l'autre et selon les périodes: en 1907, ils forment $47 \%$ du corps étudiant à l'Université de Liège, $19 \%$ à l'Université libre de Bruxelles et $23 \%$ à l'Université de Gand; en 1924, ils formeront respectivement $40 \%, 8 \%$ et $35 \%$; et en 1930, $28 \%$ à Liège, $22 \%$ dans la capitale et $34 \%$ à Gand ${ }^{55}$. La proportion d'étudiants étrangers originaires d'Europe centrale et orientale augmente de manière exponentielle entre la fin du XIX $\mathrm{X}^{\mathrm{e}}$ siècle et les années trente, passant de 14\% pour 1876-1880 à $62 \%$ en 1906-1910, et enfin $67 \%$ pour la tranche 1926193056. Ces étrangers de diverses nationalités se rassemblent et s'organisent, ils ne se contentent pas de relations informelles. Les étudiants brésiliens créent leur première association à Gand dès 1875, les Bulgares s'associent à Liège avant la Première Guerre mondiale, et dans l'entre-deux-guerres, on compte des associations d'étudiants chinois, costariciens, grecs, macédoniens, lituaniens et polonais ${ }^{57}$. La création d'associations estudiantines basées sur le pays d'origine, l'origine ethnique ou la religion n'est donc pas propre aux Juifs qui, eux aussi, se replient et créent leurs propres organisations ${ }^{58}$.

\footnotetext{
${ }^{55}$ P. Dhondt, op. cit. Établir des statistiques concernant les étudiants étrangers n'est pas simple, considérant qu'en dehors des registres universitaires, de nombreux étudiants s'inscrivaient comme élèves libres ou pour des cours isolés et ne sont pas toujours repris dans lesdits registres imprimés. Leur nombre réel serait donc plus élevé.

${ }^{56}$ P. Dhondt, op. cit., p. 37.

${ }^{57}$ E. Stols, «Les étudiants brésiliens en Belgique (1817-1914) », dans Revista de Historia, $\mathbf{n}^{\circ} 100$, Sao-Paulo, 1974, pp. 653-692. J. Hulewicz, « Studia Polaków w uniwersytecie w Liège w latach 1880-1914 », Zeszyty Naukowe Uniwersytetu Jagiellonskiego, CCXXVII, Prace Historyczne, Zeszyt 28, Kraków (1969), pp. 5-49 ; A. Despy-Meyer, « Les étudiants bulgares dans les deux universités de Bruxelles de 1870 à 1919 », dans Revue des Archives de Sofia, n 52, 1986, pp. 1-15; A. Van Acker, Slavische Studenten aan de RUG (1855-1914). Een evocatie, Gent, Archief RUG, 1984 ; P. Dhondt, op. cit.

${ }^{58}$ Ce phénomène s'observe également en France, en Allemagne et en Suisse. Pour la France, voir C. Barrera, op. cit., pp. 157-164.
} 
Comme l'a démontré Benjamin Nathans, les étudiants juifs créèrent leurs propres associations lorsqu'ils en ressentaient le besoin et n'étaient pas pleinement acceptés par les étudiants non juifs ${ }^{59}$. De nombreuses associations d'étudiants juifs furent créées et dissoutes. Au même titre que d'autres groupes étudiants, celles-ci furent souvent structurellement instables: leur nom, organisation et objectifs évoluent, leurs rapports d'activités envisagent des projets qui ne voient jamais le jour. Le nombre de leurs adhérents est difficile à estimer avec exactitude, si ce n'est pour certaines périodes et lieux, pour lesquels les sources s'avèrent plus loquaces. Par exemple, en 1925, l'Association des Étudiants juifs de Liège déclarait compter 350 membres ${ }^{60}$. Aussi, l'audience de ces associations ne peut être limitée à leurs membres, vu que leurs publications, journaux et affiches touchaient un public bien plus large. Néanmoins, comme souvent dans le monde associatif, une poignée de personnes s'affairait à la direction et à l'organisation de ces associations.

Créées au début $\mathrm{du} \mathrm{XX}^{\mathrm{e}}$ siècle, les premières associations estudiantines juives en Belgique pouvaient se compter sur les doigts d'une main. Leur nombre augmenta pendant l'entre-deux-guerres, de même que leurs dissensions et combats pour des causes variées. L'internationalisme avait conquis les membres du Cercle des Étudiants russes de l'ISCEA à Anvers avant la Première Guerre mondiale. Ceux-ci s'opposaient au sionisme promu par le Cercle des Étudiants juifs créé à Anvers en $1912^{61}$. Ce groupe comptait quarante membres et avait comme principal objectif de raffermir les liens d'amitiés entre Juifs russes étudiant à l'Institut supérieur de l'État à Anvers (ISCEA), vu leurs divisions politiques. Ce cercle s'efforçait aussi d'envisager des débats d'idées rassembleurs portant sur la condition des Juifs dans le monde, la littérature et l'art juifs et toutes les questions politiques, économiques et sociales liées à la vie juive en général. Mais, entre 1905 et 1914, Anvers n'était pas le principal pôle estudiantin juif en Belgique. Liège dominait et connaissait également l'opposition entre étudiants sionistes et non sionistes.

${ }^{59}$ B. Nathans, Beyond the Pale. The Jewish Encounter with Late Imperial Russia, Berkeley - Los Angeles, 2002, pp. 245-260.

${ }^{60}$ R. Van Doorslaer, op. cit., p. 86.

${ }^{61}$ V. Ronin, Antwerpen en zijn 'Russen': onderdanen van de tsar, 1814-1914, Gand, 1993, pp. 174183. 
Après la Première Guerre mondiale, sur base des associations existant avant-guerre et avec l'aide d'une communauté juive active et en pleine expansion, de nouvelles associations virent le jour. Ce fut le cas notamment de Funck, l'Union des Étudiants juifs de Gand, groupe s'identifiant à la culture et à la langue yiddish. Les membres de cette association devaient remplir les conditions suivantes: «(a) Être ou avoir été étudiant(ante) à Gand; (b) reconnaître la langue Idysch comme seule langue des masses juives; (c) reconnaître le présent statut. " ${ }^{62}$ Funck s'opposait à l'Association des Étudiants juifs de Gand, de tendance sioniste, qui avait élu en son sein un secrétaire pour l'hébreu et un pour le yiddish et mentionnait dans l'en-tête de son courrier le nom de l'association en hébreu, en yiddish et en français ${ }^{63}$.

Une tentative de regrouper les diverses organisations estudiantines juives vit le jour avec la création de la Fédération des Associations d'Étudiants juifs de Belgique (FEJB), dirigée par Julien J. Grunberg. Cette organisation faîtière regroupait notamment Heatid, l'Association des Étudiants juifs d'Anvers, le Cercle des Étudiants juifs de Bruxelles, l'Association des Étudiants juifs de Gand et l'Association des Étudiants juifs de Liège ${ }^{64}$. Les réunions de l'organisation se tenaient successivement dans l'une des quatre villes où la FEJB était présente. Les délégués s'y rendaient en train et se réunissaient généralement dans un café près de la gare, comme le Café Paon Royal sur la place de la Gare à Anvers 65.

La FEJB décida de soutenir et de s'affilier à l'Union mondiale des Étudiants juifs (UMEJ) dès la création de cette association en 1924. L'enthousiasme fut tel que, sous l'impulsion de Grunberg, la FEJB proposa d'organiser le premier congrès mondial de l'UMEJ à Anvers, avec le soutien de la Fédération sioniste de Belgique et du secteur diamantaire $^{66}$. Celui-ci s'y tint du 30 avril au 4 mai 1924, comme il ressort des illustrations 3 et 4. Créée par Zvi Lauterpach, un étudiant

${ }^{62}$ Archives de l'Université de Gand (AUG), Kabinet van de Rector, 424, 107, 1922-1923, 323 b Studentenverenigingen.

${ }^{63}$ Ibid.

${ }^{64}$ Archives du YIVO à New York (YIVO), Collection David Trocki, 34.

65 YIVO, Collection David Trocki, 34, Réunion de la Fédération des Étudiants juifs de Belgique qui eut lieu à Anvers le dimanche 9 octobre à $9 \mathrm{~h} 30$.

${ }^{66}$ D. Dratwa - Z. Seewald, Avis à la Population. L'histoire juive s'affiche. Public Notice. Jewish History in Posters, Musée juif de Belgique, Bruxelles, 2010, p. 48. 
autrichien, l'UMEJ séduisit de nombreux intellectuels, qui s'allièrent pour lutter contre les numerus clausus et quotas imposés aux Juifs à l'entrée dans les universités et établissements d'enseignement supérieur. Parmi ces intellectuels figurait Albert Einstein, qui devint le premier président de l'association en $1925^{67}$. Au sein du comité soutenant l'association, on trouvait notamment le poète Chaïm Nachman Bialik, l'historien Simon Dubnow et Sigmund Freud ${ }^{68}$. L'une des priorités de l'UMEJ était de protéger ses membres; l'organisation proclamait incarner les étudiants juifs unifiés ${ }^{69}$. L'UMEJ prit part à diverses conférences et congrès internationaux pour $\mathrm{y}$ plaider la cause des étudiants juifs.

\section{Les associations d'étudiants juifs en Belgique}

Au-delà des fonctions de représentant et porte-parole des étudiants auprès des autorités académiques et étatiques, l'objet principal des associations d'étudiants juifs en Belgique était de porter secours aux étudiants démunis. L'ingéniosité était de mise, les idées ne manquaient guère : ils organisaient des bals, collectaient de l'argent auprès des diamantaires anversois et au sein de l'establishment juif à Bruxelles. La collecte ne se limitait pas à l'espace national : ils plaidèrent leur cause au-delà des frontières et frappèrent à la porte de l'Alliance israélite universelle (AIU) à Paris et au Joint Distribution Committee (JDC) à New York. En 1926, le rapport de la FEJB indique que son objectif principal est de créer un Fonds central d'Assistance économique afin de centraliser toutes les informations concernant les étudiants nécessiteux et les soutiens financiers ${ }^{70}$. L'organisation projeta aussi de mieux se préparer en vue de futures négociations avec les autorités académiques concernant la question de la reconnaissance des diplômes et des délais de payement des frais d'inscription.

\footnotetext{
${ }^{67}$ J. Voloj, op. cit., p. 75.

68 YIVO, Collection David Trocki, 34, Annexe 1, Rapport sur l'activité de l'Union mondiale, avril 1925.

${ }^{69}$ Léo Steinig, General Secretary of the World Union of Jewish Students (WUJS), « Partie officielle. Dans l'Union mondiale», dans Bulletin de la Fédération des Étudiants juifs de Belgique, janvier $1925,1^{\mathrm{c}}$ année, $\mathrm{n}^{\circ} 1$, p. 25.

${ }^{70}$ YIVO, Collection David Trocki, 34, Lettre du 2 avril 1926.
} 
Néanmoins, malgré ses contacts internationaux et ses projets intéressants, la FEJB pâtit de son statut d'organisation faîtière dépendante des associations la composant. Le nombre de ses membres déclina et ses branches locales prêtaient peu d'attention aux activités nationales. Il faut toutefois noter que deux associations qui virent le jour dans des villes de taille moyenne furent reconnues et intégrées à l'organisation : il s'agit du Cercle des Étudiants juifs de Gembloux et du Groupe des Étudiants sionistes de Verviers. Elles furent portées par les étudiants juifs d'Europe centrale et orientale inscrits à l'Institut agricole de l'État à Gembloux et à l'École supérieure des Textiles à Verviers.

Une association dynamique fut créée à Bruxelles en 1927-28, l'Association des Étudiants Juifs de Bruxelles (AEJB), avec comme but premier de constituer un lieu de rencontre pour les étudiants juifs originaires de Pologne, de Roumanie, et d'autres pays où ils étaient victimes de persécutions ${ }^{71}$. Cette association visait à procurer un soutien moral, culturel, financier et juridique à ses membres. Tout comme le fit la FEJB, l'AEJB publia des tracts et même ses cartes de membres en trois langues: hébreu, yiddish et français [Illustration 5], soulignant de ce fait la prépondérance sioniste mais aussi le respect des yiddishistes.

Se proclamant apolitique, ce groupe organisait de nombreuses conférences scientifiques et littéraires, un bal annuel, et mettait à disposition de ses membres une petite bibliothèque ainsi qu'une caisse de soutien. La plupart de ses ressources financières provenait des frais d'inscription, de la participation à la loterie et autres activités philanthropiques comme le bal annuel. L'AEJB avait pignon sur rue, ses bureaux étaient situés rue Saint-Boniface à Ixelles, au cœur du quartier latin des étudiants de l'ULB. Visibilité et prestige étaient de mise. Ils avaient coutume d'organiser leur bal annuel «au Palais d'Egmont, la plus belle salle de Bruxelles ", selon Chana Zosia Perelman $^{72}$. Si l'on se réfère aux quelques lignes mentionnant l'AEJB dans Bruxelles universitaire, l'un des principaux journaux estudiantins de l'ULB, l'association fut créée dans le but d'accueillir les étudiants

71 YIVO Archives, Collection David Trocki, 34

72 IEJ, Interview de Sophie Schneebalg-Perelman par Daniel Dratwa, Bruxelles, 18 avril 1981. 
juifs étrangers "venus de toutes les parties du monde " $^{73}$, en vue de «fournir [à ses membres] tous les renseignements nécessaires pour se débrouiller dans leur nouvelle vie d'étudiant à l'étranger » ${ }^{74}$. En 1935 , l'affiche du bal annuel est rédigée presque exclusivement en français, ce qui révèle la volonté de s'affirmer comme une association intégrée à la vie associative locale, dont les membres comprennent la langue du pays [Illustration 6].

Si de nombreuses associations estudiantines furent créées puis rapidement dissoutes, elles contribuèrent néanmoins à entretenir le dynamisme de la vie culturelle et sociale juive en Belgique. Les archives de la collection David Trocki reflètent cet essor. On y trouve maintes affiches, dépliants, appels à se rassembler, à participer aux fêtes, bals et conférences organisées par quelques dizaines d'organisations. Ces documents illustrent la diversité de la communauté juive immigrée. Les affiches sont publiées en plusieurs langues: en français, en néerlandais, mais aussi en hébreu et en yiddish, et souvent un même événement est annoncé en plusieurs langues, comme le montrent des affiches [Illustrations 7 et 8 ], pour un bal étudiant coorganisé par Heatid, l'Association des Étudiants juifs d'Anvers et les Anciens Combattants juifs polonais.

La plupart des associations ne pouvaient se permettre de louer des bureaux, comme l'AEJB. Les membres devaient se contenter de salles de réunions à l'arrière de cafés et de restaurants. Chaque organisation avait son QG : l'Association des Étudiants juifs de Gand opta pour le restaurant Kousminsrey, alors que Funck, les yiddishistes, choisit le Café des Notaires, rue de Flandre ${ }^{75}$. L'importance des cafés pour la sociabilité des étudiants russes en Allemagne a été soulignée par Claudie Weill, qui distingue les cafés des restaurants, qui constituaient fréquemment un lieu de confrontation entre étudiants étrangers et nationaux ${ }^{76}$. Nous n'avons pas trouvé trace d'une distinction et d'affrontements semblables en Belgique.

Les associations d'étudiants juifs en Belgique se composaient majoritairement de Juifs d'Europe centrale et orientale. Selon Paul

\footnotetext{
73 AULB, A. B., « Association des Étudiants juifs à Bruxelles », dans Bruxelles universitaire numéro spécial St-Verhaegen, 10e année, 1928.

74 Ibid.

${ }^{75}$ AUG, 4 A 24, Kabinet van de Rector, 235, 1930-1931, boîte 323.

${ }^{76}$ C. Weill, op. cit., p. 195.
} 
Lévy, au début des années 1930, 40 à $50 \%$ des membres de ces associations venaient de Pologne, puis en ordre descendant ils étaient originaires de Roumanie, de Palestine et des Pays baltes ${ }^{77}$. Le nombre des membres de l'AEJB diminua et passa de 180 en 1933 à 110 en 1938, suite aux restrictions migratoires. À la fin des années 1930, seuls $10 \%$ des membres de l'AEJB possédaient la nationalité belge, les autres étant originaires de Pologne, Allemagne, Autriche, Roumanie, mais aussi de Lettonie, Lituanie, Palestine et des États-Unis. Ils se spécialisaient en sciences, optaient pour les sciences appliquées, la médecine, la chimie, la pharmacie ou encore l'ingénierie ${ }^{78}$. La proportion entre étudiants hommes et femmes parmi les membres des associations juives penchait comme on peut s'y attendre en faveur des hommes, vu le faible nombre d'étudiantes dans l'absolu d'une part et leur participation généralement réduite dans le monde associatif estudiantin d'autre part. Au sein du comité de l'Association des Étudiants juifs de Gand par exemple, sur les quatorze membres composant le comité en 1931, on ne trouvait qu'une seule femme ${ }^{79}$.

Quelle place réservaient aux Juifs nés en Belgique ces associations composées majoritairement d'étudiants étrangers ? Lévy, immigré de seconde génération, critique ces associations qui prétendaient accueillir tous les étudiants juifs sans distinction mais en pratique excluaient les Belges, excepté quelques étudiants dont les parents étaient venus d'Europe de l'Est. Cette référence à son propre cas illustre fort probablement son expérience personnelle. Vu comme juif dans les groupes non juifs, il était rejeté et perçu comme «le Belge » dans les associations juives dominées par les immigrés.

\section{Ambivalences et stéréotypes}

Les cercles étudiants et associations diverses, de même que les salles de cours, salles de fêtes et terrains de football sont des lieux d'interaction entre étudiants belges et étrangers. Mais quelle est la nature de leurs rapports ? Comment se perçoivent-ils mutuellement?

77 P. M.-G. Lévy, « Les Étudiants étrangers en Belgique », dans Revue du Cercle des Alumni de la Fondation universitaire, vol. 4, 1, Bruxelles, octobre 1932, pp. 30-31.

${ }^{78}$ CCIB, Archives de Moscou, 160.1.10, Lettre non datée.

${ }^{79}$ AUG, 4 A24 Kabinet van de Rector, 235, 1930-1931, boîte 323. 
Quels stéréotypes perdurent? De manière concrète, que se disent-ils ? Quand débattent-ils et de quelle manière flirtent-ils, pour autant que de tels contacts aient eu lieu ? Enfin, les lignes qui suivent souligneront les différences existant entre les étudiantes juives d'Europe centrale et orientale et leurs camarades belges, en termes d'implication dans la vie sociale universitaire et de relations aux étudiants du sexe opposé. Ceci nous permettra de cibler les clichés visant spécifiquement les étudiantes juives de l'Est sur base de leur genre et de leur ethnicité.

À l'aube du XX' $\mathrm{XX}^{\mathrm{e}}$ siècle, selon Bernadette Lacomble-Masereel, qui publia une étude remarquable sur les étudiantes à l'Université de Liège, les étudiantes juives de l'Empire tsariste se comportaient différemment de leurs camarades belges. Elle relate ainsi que ces dernières « ont pris une part active aux bals, aux soirées et même aux réunions politiques organisées par la colonie russe $»^{80}$. Elles s'engagèrent plus que les Belges dans des groupes politiques, participaient aux réunions, prenaient la parole en public. Aussi, les étudiantes étrangères se mêlaient bien plus que les Belges aux étudiants du sexe dit fort ${ }^{81}$. Les causes de ces différences sont à chercher notamment dans leur écart d'âge. Les étudiantes de l'Est sont plus âgées que leurs consœurs. Elles sont également plus mûres, ayant émigré seules, quitté leur famille, pays et culture et devant faire preuve de ressources pour affronter l'inconnu. De plus, les étudiantes migrantes sont libres, ayant échappé, du moins en partie, à la pression sociale et familiale, habitant seules ou avec d'autres étudiantes. Elles ne sont plus soumises qu'au regard de leurs logeuses, qui d'ailleurs souvent les méprisent et critiquent leur prétendu libertinage. Néanmoins, en dépit de cette liberté relative, les étudiantes venues d'ailleurs n'usent pas comme elles le souhaitent de leur temps libre. Elles sont souvent contraintes, pour des raisons financières, à travailler en sus de leurs études et n'ont que peu ou pas d'argent à consacrer aux tenues et robes pour soirées dansantes, et pas de temps pour assister aux conférences ${ }^{82}$. Enfin, une différence importante également entre les

\footnotetext{
${ }^{80}$ B. Lacomble-Masereel, op. cit., p. 54.

${ }^{81}$ Ibid., p. 173.

${ }^{82}$ Sur la situation matérielle des étudiants juifs de l'Est, voir P. Falek, « Travailler ou étudier : là n'est pas la question. Analyse des modes de subsistance d'étudiants juifs d'Europe de l'Est en Belgique (1918-1940) », dans Les Cahiers du Judaïsme, n 29, Paris, 2010, pp. 32-43.
} 
étrangères et les Belges est leur degré d'intérêt pour la chose politique. Nous y reviendrons.

Pendant l'entre-deux-guerres, de nombreuses activités culturelles, sociales et politiques furent organisées par les associations d'étudiants juifs. Un étudiant non juif, membre de la Société générale des Étudiants libéraux de l'ISCEA, relate ainsi s'être bien amusé lors d'une fête chapeautée par Heatid, l'Association des Étudiants juifs de l'ISCEA, en février 1924. Il raconte ce qui suit: « Nous avions chaud, serrés sur un banc, et si près de tant de si belles, si délicieuses, si captivantes, si enchanteresses, petites Juives. Un bal à la hauteur clôtura cette soirée si réussie - j'ai pu serrer dans mes bras ces charmantes petites Èves... ${ }^{83}$ Cet extrait est intéressant pour trois raisons. Premièrement, il confirme la présence d'étudiantes juives de l'Est sur les pistes de danse. Deuxièmement, il atteste de l'interaction entre étudiants juifs et non juifs. Enfin, ce texte révèle la persistance parmi les étudiants belges de vieux stéréotypes antisémites, selon lesquels la femme juive serait une créature aussi séduisante que dangereuse.

Les discriminations visant les étudiantes, belges et étrangères, ne sont pas propres au monde universitaire belge. Comme le soulignent Irina et Dimitri Gouzevitch, elles sont également présentes chez nos voisins français : « La situation des étudiantes étrangères est encore plus difficile, car elles doivent affronter non seulement les moqueries et les brimades des étudiants mais également le comportement méprisant de certains professeurs, qui refusent de les prendre au sérieux. $»^{84}$

Les étudiantes juives d'Europe centrale et orientale étaient fréquemment l'objet de moqueries. Bruxelles universitaire rapporte ainsi qu'en mars 1930, un plaisantin de Charleroi suggéra de créer un « Pogrom's Club » visant à persécuter « des étudiants juifs et surtout des plumes juives, fort jolies ${ }^{85}$. Le texte précise également que cet étudiant criait sur tous les toits : «Moitié bistro, moitié ghetto. Telle est la première philo. ${ }^{86}$ L'auteur de l'article ajoute néanmoins que

${ }^{83}$ "Le Festival du Cercle "Heatid" ", dans Le Flux. Organe officiel de la Société générale des Étudiants libéraux de l'ISCEA, n 3, 24 février 1924, p. 5.

${ }^{84}$ D. et I. Gouzevitch, «Étudiants, savants et ingénieurs juifs originaires de l'Empire russe en France (1860-1940) », dans Archives juives, vol. 35/1, 2002, pp. 123-124.

${ }^{85}$ AULB, « Un Pogrom's club », dans Bruxelles universitaire, mars 1930, p. 8.

${ }^{86}$ Ibid. 
cette blague peut être considérée comme un non-événement. Des étudiants juifs s'étant « émus outre mesure », le « Pogrom's Club » n’a jamais vu le jour. Il nous faut souligner que si, selon l'article en question, les étudiantes juives «envahissaient» la Faculté de Philosophie et Lettres, elles étaient en réalité deux fois plus nombreuses dans les Facultés de Sciences naturelles et de Médecine.

Usant également de références antisémites mais sans prétendre cette fois à la drôlerie, d'autres articles s'accordèrent pour demander, sur le modèle hongrois ou polonais, un numerus clausus pour les étudiants étrangers ${ }^{87}$. Publiés en 1925 dans l'Étudiant catholique, organe des Étudiants catholiques de Gand, et dans L'Étudiant libéral, journal des Étudiants libéraux de l'Université de Liège, ces articles exigeaient d'opérer comme pour l'affaire des corps ${ }^{88}$ dans les facultés de médecine en Pologne : «Comme le nombre de cadavres est fort petit, on a intérêt à ne pas augmenter celui de ceux [sic] qui doivent les étudier. " $^{99}$ Les étudiants juifs critiquèrent avec véhémence ces positions et arguèrent qu'elles émanaient d'étudiants polonais et roumains non juifs qui tentaient d'importer l'animosité propre à leurs pays d'origine. Julien Vermont, auteur de l'article en question, ne pouvait croire que des étudiants belges, toujours si accueillants envers les Juifs, pouvaient répandre de telles infamies. Encensant les universités belges comme autant d'espaces d'ouverture, il conclut comme suit : « Là où la bonne et franche camaraderie a toujours existé entre Belges et étrangers, entre chrétiens et juifs, entre gens d'opinions contraires et de confessions diverses, il n'y a pas de place pour les fauteurs de désordres. ${ }^{90} \mathrm{La}$ position de Julien Vermont, étudiant juif belge, fait écho à celle de Paul M.-G. Lévy qui, lui aussi, tenta d'embellir l'image des relations entre étudiants juifs et belges de souche.

\footnotetext{
87 J. Vermont, "Un nouvel article d'importation. Le Numerus Clausus », dans Bulletin de la Fédération des Étudiants juifs de Belgique, $1^{\text {ère }}$ année, ${ }^{\circ}$ 4, mai 1925, pp. 130-133.

${ }^{88}$ N. Aleksiun, "Christian Corpses for Christians ! Dissecting the Anti-Semitism behind the Cadaver Affair of the Second Polish Republic », dans East European Politics and Societies, 2011, vol. $25, \mathbf{n}^{\circ} 3$, pp. $393-409$.

89 “Numerus Clausus", dans L'Étudiant catholique, avril 1925, p. 2. Cité par Julien Vermont, op. cit.

${ }^{90}$ J. Vermont, op. cit., p. 133.
} 
Selon Paul M.-G. Lévy, la xénophobie n'existait pas dans les universités belges ${ }^{91}$. Lévy plaida la cause des étudiants étrangers dans une réponse à un article paru dans La Flandre libérale présentant les étudiants étrangers comme autant de concurrents des diplômés locaux. Des «concurrents » étrangers, telle était la représentation propagée par de nombreux étudiants, professeurs et professionnels français quand ils décrivaient les étudiants de l'Est, et ce surtout dans les années 1930. Les réactions antisémites s'expriment de manière plus ou moins subtile et sont généralement liées, dans le cas français, à l'accès à la profession ${ }^{92}$. La Belgique semble plus accueillante et ne connut pas de mobilisation menant à légiférer sur l'exercice de certaines professions, comme ce fut le cas en France avec la loi Armbuster du 21 avril 1933, limitant l'accès à la médecine aux diplômés nationalisés français ${ }^{93}$. Semblables critiques aux relents antisémites existaient en Belgique, mais restaient limitées à certains organes de presse.

\section{Les préoccupations des étudiants étrangers}

Pour Lévy, ingénieur juif de nationalité belge, immigré de seconde génération, les relations entre étudiants belges et étrangerscomprenons: Juifs d'Europe de l'Est - étaient excellentes. La principale singularité de ces derniers résidait dans leur sens de l'humour, dans la capacité plus grande des étrangers à alléger leurs soucis par la dérision. Mais, selon Lévy, les visions divergentes des étudiants belges et étrangers s'enrichissent de leur complémentarité, ce qui contribue à améliorer les résultats de leur collaboration quotidienne ${ }^{94}$. L'une des différences frappantes entre étudiants belges et Européens de l'Est se situe dans leur rapport à l'étude, les seconds témoignant respect et considération aux professeurs et à la Science. La nature des relations décrites par Lévy comme cordiales, voire amicales, entre étudiants belges et étrangers doit être nuancée. Pour les

${ }^{91}$ P. M.-G. Lévy, op. cit., p. 29.

92 C. Barrera, op. cit., pp. 148-150.

${ }_{93}$ N. Manitakis, « Étudiants étrangers, universités françaises et marché du travail intellectuel (fin du $\mathrm{XIX}^{\mathrm{e}}$ - années 1930) », dans E. Guichard - G. Noiriel, Construction des nationalités et immigration dans la France contemporaine, Paris, 1997, pp. 123-154.

${ }^{94}$ P. M.-G. Lévy, « Les étudiants étrangers en Belgique », dans La Flandre Libérale, 21 décembre 1931. 
comprendre, il est nécessaire de tenir compte de la divergence des arrière-plans socio-culturels respectifs. Elie Pouterman, étudiant juif de Bessarabie à l'Université de Gand, raconte à ce sujet : "Quand je suis arrivé à Gand, j'étais très étonné. J'avais déjà lu tout Ibsen et Maeterlinck en russe. Mais les étudiants de l'Université de Gand étaient très peu au courant de la littérature mondiale. Tout ce qu'ils connaissaient, c'était un peu de littérature française de l'école. $\nu^{95}$ Les étudiants juifs d'Europe de l'Est ne comprenaient pas l'indifférence relative de leurs homologues belges envers ce qui les passionnait: la politique internationale. Les courants idéologiques de la fin du XIX siècle ont bercé les Juifs d'Europe centrale et orientale, les orientant vers le communisme, le socialisme, le sionisme, le folkisme, etc. Enfin, ces étudiants étrangers restaient tout simplement très préoccupés par la situation et l'évolution de leur pays d'origine, où nombre d'entre eux pensaient retourner et où la plupart avaient laissé des proches. Caroline Barrera explique que les étudiants étrangers inscrits à la Faculté de Droit de Toulouse étaient sensibles à la situation internationale pour plusieurs raisons : "Eux-mêmes sont réfugiés et donc vivent dans des conditions matérielles et psychologiques qui ne sont pas propices à l'étude. [...] Ils sont occupés par des activités plus immédiatement importantes (survie, aide aux réfugiés et activité politique). [...] Ils sont mobilisés et donc doivent interrompre leurs études. $\nu^{96}$

Malgré les arguments de Lévy, il semble que bien des étudiants étrangers se soient repliés sur eux-mêmes, créant leurs propres associations, parlant leur langue et ainsi s'auto-excluant. La méconnaissance de la langue française est l'une des causes majeures de ce repli. Malgré la nécessité théorique de posséder la langue française avant d'immigrer, la majorité des étudiants l'apprennent sur le tas, à l'aide d'un dictionnaire. Alain Berenboom raconte que son père, Chaïm Berenbaum, ne connaissait pas un mot de français lorsqu'il arriva en 1927 à Liège pour y étudier la pharmacie ${ }^{97}$ : «Pour apprendre le

95 AUG, interview d'Elie Pouterman, par Anne-Marie Simon Van der Meersch, 1981.

${ }^{96}$ C. Barrera, op. cit., p. 123.

${ }^{97}$ Chaïm Berenbaum et ses deux sœurs, Esther (née en 1912) et Sura Hendla Berenbaum (née en 1910), nés à Maków Mazowiecki, sont tous trois venus étudier en Belgique, les deux premiers la pharmacie, tandis que Sura Hendla suivit un cours isolé en Histoire de la Littérature française en Faculté de Philosophie et Lettres à l'ULB en 1937-38. Considérant qu'elle ne faisait pas d'études 
français, il lisait tous les jours le journal à haute voix à ses copains de chambrée. ${ }^{98}$ Quelques étudiants s'inscrivent en langue et littérature françaises sans pour autant lire ou parler le français, comme l'a révélé Etlea Coperbac des années plus tard ${ }^{99}$. Coperbac, née à Kichinev en 1909, rejoint sa sœur en Belgique en 1938 et s'inscrit à l'ULB'100. Elle garda toute sa vie un accent slave très prononcé en français, langue qu'elle ne maîtrisa jamais, comme on peut l'entendre dans son interview. La langue constituant un obstacle, les étudiants préfèrent se retrouver avec leurs compatriotes et coreligionnaires, avec lesquels ils s'entretiendront en polonais ou en yiddish. Fela Perelman-Liwer relate ainsi dans un entretien : « $\grave{A}$ part quelques amis intimes [...], je n'ai pas beaucoup fréquenté les milieux autres que ceux des étudiants étrangers. »101 Elle a rejoint «naturellement » l'association des étudiants juifs où se réunissaient de nombreux étudiants polonais et roumains. Elle raconte n'avoir pas cherché à rencontrer des étudiants belges, préférant la compagnie de ceux dont elle partageait l'idiome.

L'image donnée par les associations étudiantes juives était celle du mystère qui engendre la suspicion, entraînant le rejet et par conséquent le repli sur soi des étudiants étrangers. Les étudiants juifs de l'Est, isolés, parlant entre eux le yiddish, étaient perçus comme formant une communauté fermée et secrète ${ }^{102}$. Les représentants de ces associations tentèrent de modifier cette image et de démontrer son inexactitude. La FEJB justifia son existence et celles d'autres associations d'étudiants juifs de la sorte : "Parmi les étudiants juifs, ressortissant de plusieurs pays, se manifeste une certaine communauté

sérieuses, son père lui imposa de rentrer en Pologne, ce qu'elle fit en février 1940. Elle mourut en déportation. Esther entama à l'Université de Liège en 1933 des études de pharmacie qu'elle termina à l'ULB en 1939. Les deux sœurs étaient soutenues financièrement par leur frère Chaïm, arrivé en 1927. AUL (Archives de l'Université de Liège), Registre des étudiants, carte d'étudiant de Chaïm Berenbaum et d'Esther Berenbaum ; AULB, Registre des étudiants, carte d'étudiante d'Esther Berenbaum; Archives de la Commune de Schaerbeek, Registre des Étrangers, Dossiers individuels, Dossier de Sura Hendla Berenbaum A. 229.962. Discussion avec Marc Goltzberg, fils d'Esther Berenbaum et de Mosco (Michel) Goltzberg, Bruxelles, février 2011. Discussion avec Alain Berenboom, fils de Chaïm Berenbaum, Bruxelles, juin-juillet 2011.

${ }^{98}$ A. Berenboom, Le Fils préféré du magicien, Bruxelles, 2011, tapuscrit inédit, p. 40.

${ }^{99}$ Archives privées de Claire Van Loo ; interview d'Etlea Coperbac par Claire Van Loo, Bruxelles, 29-30 décembre 1985, cassette 1 .

${ }^{100}$ AULB, Registre des étudiants, carte d'étudiante d'Etlea Coperbac.

${ }^{101}$ FMC, interview de Fela Perelman, par J.-Ph. Schreiber, Bruxelles, 1984, pp. 13-15.

102 YIVO Archives, Collection David Trocki, 34, Annexe 2. 
d'intérêts qui les pousse à se tenir ensemble et à s'organiser, et une cohésion suffisante pour que cette organisation soit viable, favorisée qu'elle était par la circonstance que la quasi-totalité des étudiants juifs en Belgique sont ici en pays étranger. Ceci explique la création spontanée dans les villes universitaires des organisations générales des étudiants juifs, ouvertes à tous, sans distinction de pays, de provenance ou de couleur d'opinion et ayant pour but d'améliorer la situation morale et matérielle des étudiants juifs, les représenter le cas échéant, etc. ${ }^{103}$ Malgré ces explications, les associations étudiantes juives restèrent perçues comme mystérieuses et surtout comme des refuges pour communistes et révolutionnaires en tous genres.

\section{Conclusion}

À l'aube du $\mathrm{XX}^{\mathrm{e}}$ siècle, en avance sur leur temps, émancipées et libres de toute pression familiale, les étudiantes juives venues de l'Est s'engagent corps et âme, bien plus que leurs consœurs belges, dans des groupes culturels, sociaux et politiques. Or les cercles étudiants restent un monde d'hommes, où fumer et boire constituent la pierre angulaire et où les femmes ne sont pas les bienvenues. Néanmoins, petit à petit, à défaut d'entrer dans les cercles facultaires, les femmes créent leurs propres associations et s'engagent, aux côtés des hommes, dans des groupes politiques, sportifs et culturels. Mais en plus d'être femmes, leur ethnicité et leur nationalité entrent en ligne de compte. Les étrangers sont, en théorie, et selon de nombreux mémorialistes contemporains, bien accueillis par les étudiants belges. Et pourtant, ils ressentent le besoin de former leurs propres associations et donc d'adopter une attitude de repli. Les étudiants juifs d'Europe centrale et orientale ne sont pas en reste. Ils créent plusieurs associations suivant les lignes politiques et les idéologies ayant le vent en poupe, regroupées par moment mais sans grand succès, permettant toutefois quelques éclatantes réussites, comme d'organiser le Premier Congrès mondial des Étudiants juifs à Anvers en 1924. Ces associations, qui polarisent les besoins des étudiants, servent de catalyseurs à leurs revendications. Elles leur permettent par ailleurs de rassembler les

${ }^{103}$ YIVO Archives, Collection David Trocki, 34. 
fonds nécessaires à aider les plus démunis et favorisent par là même la solidarité et la fraternité estudiantines. Se retrouver avec ses compatriotes ou d'autres personnes parlant une langue commune et partageant la même culture était par ailleurs, et est toujours si l'on observe les étudiants étrangers aujourd'hui, rassurant pour ces jeunes immigrés, la tendance à l'isolement étant pour eux d'autant plus prononcée qu'ils pouvaient s'insérer dans une communauté d'expatriés dont le dynamisme culturel allait croissant.

Le degré d'intégration et de socialisation des étudiantes dépend également de facteurs inhérents à leur projet migratoire. La durée de leur séjour et le désir de regagner leurs pays natal ou d'émigrer en Palestine ou en Amérique influenceront leur attitude par rapport aux étudiants belges. La connaissance de la langue française, prérequis théoriquement obligatoire à l'immigration estudiantine, jouera également un rôle fondamental dans leur processus d'adaptation, cet apprentissage se faisant souvent une fois les intéressées arrivées en Belgique.

Enfin et pour conclure, l'intégration paradoxale des étudiantes juives immigrées de deuxième génération est à souligner. Ce sont elles les véritables agentes de l'interaction entre étudiants juifs et non juifs au sein de l'Université, par leur implication dans l'organisation des associations culturelles. Ce qui n'empêche qu'elles se voient cantonner à une position marginale et sont tributaires du processus de repli des étudiants juifs migrants en provenance d'Europe centrale et orientale. Et ce en dépit du fait que ces associations ne manquent pas de publier leurs affiches et leurs tracts en plusieurs langues et se proclament ouvertes à toutes et tous, sexe et tendance politique confondus. 
Portrait de Paul Lévy (AULB, Bruxelles universitaire, St-Verhaegen 1931, p. 20) [ill. 1].
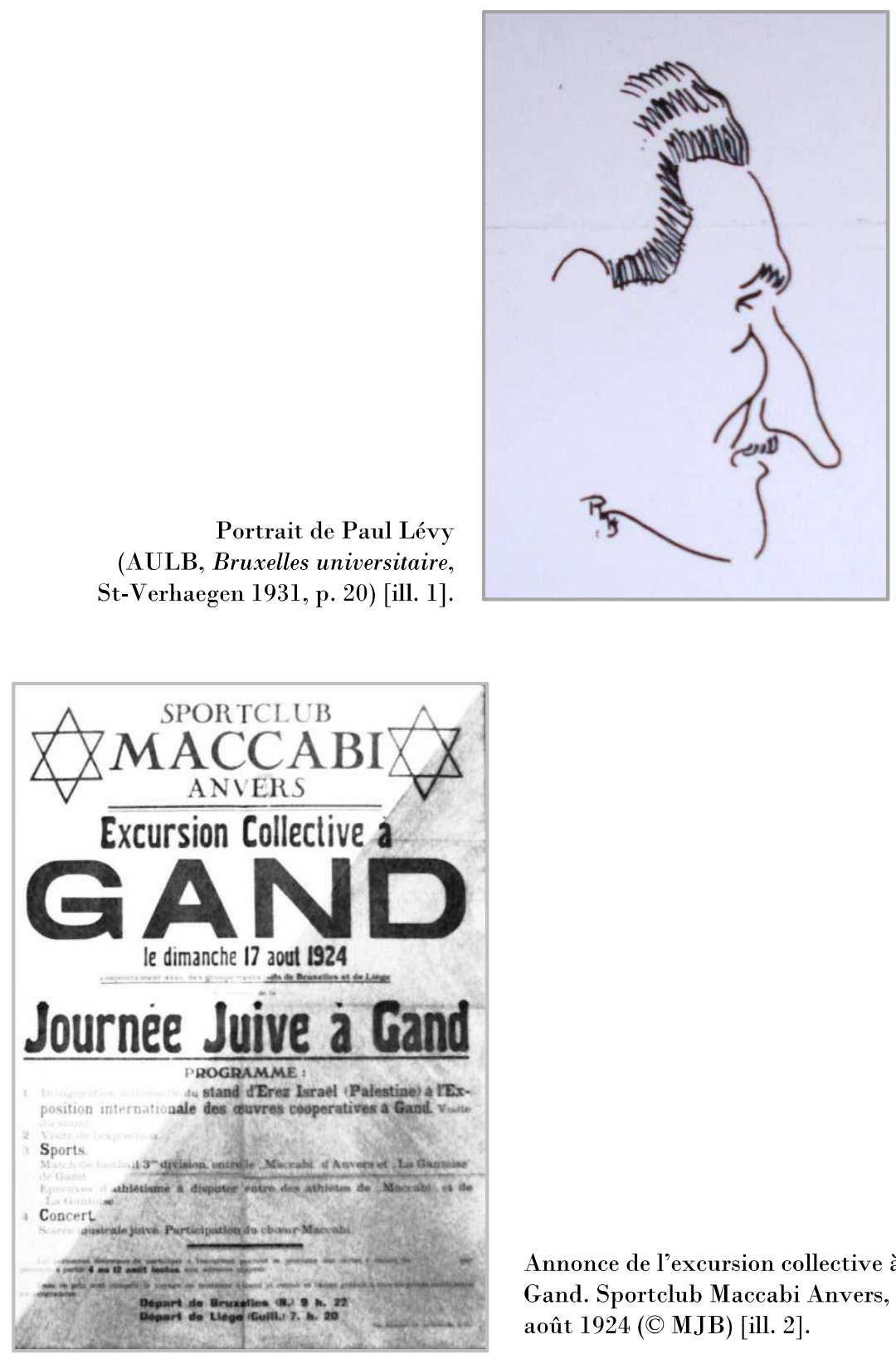

Annonce de l'excursion collective à Gand. Sportclub Maccabi Anvers, 17 août 1924 (C MJB) [ill. 2]. 


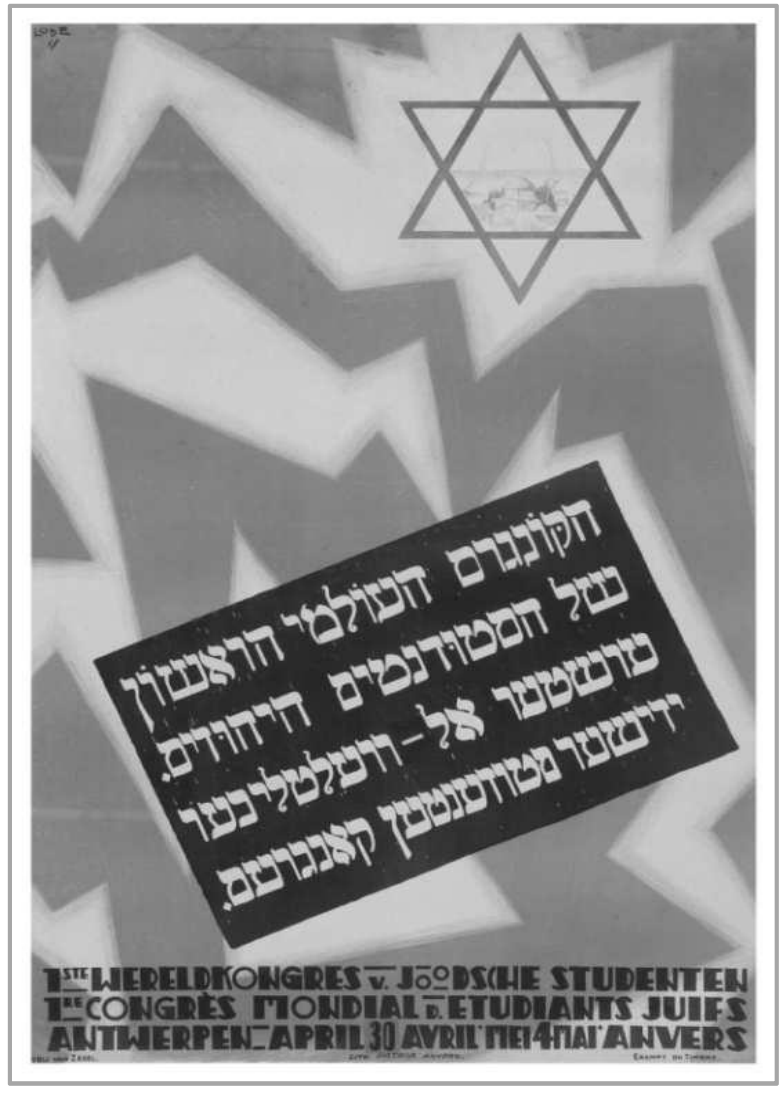

Poster du Premier Congrès mondial des Étudiants juifs, Anvers, 1924

(C) MJB) [ill. 3]. 


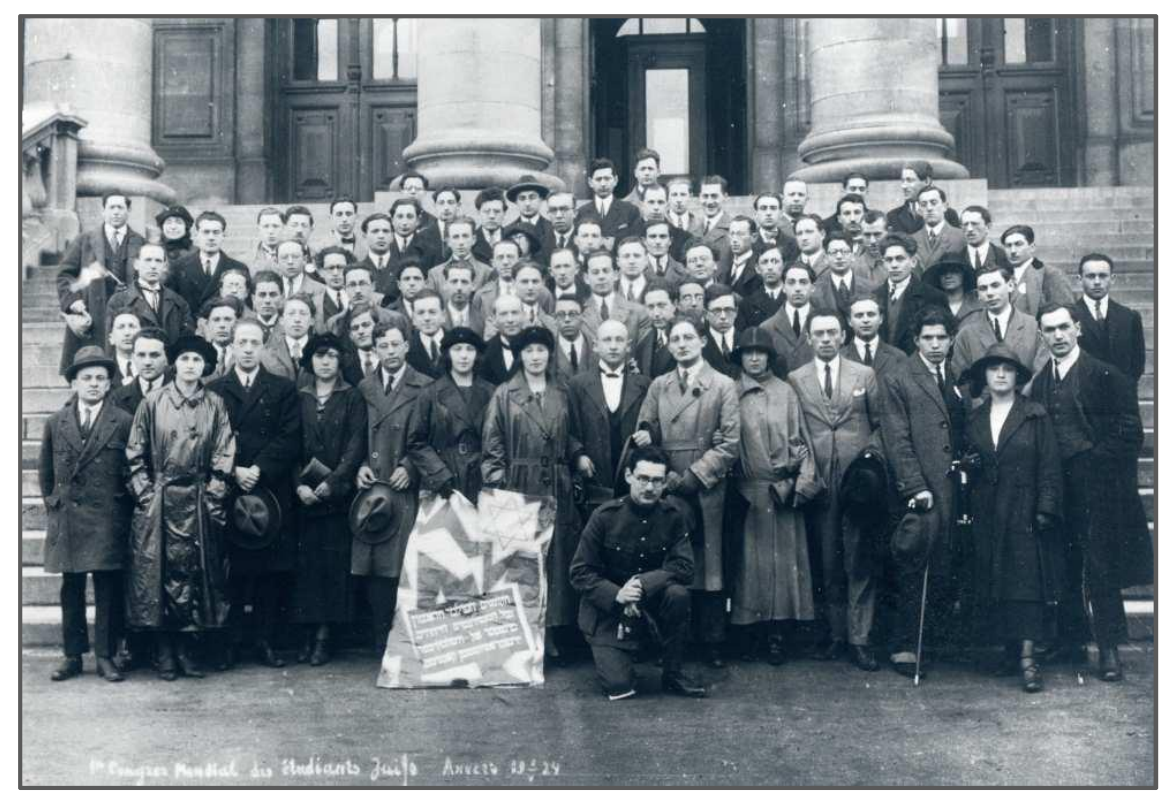

Premier Congrès mondial des Étudiants juifs, Anvers, 1924 (C) MJB) [ill. 4].

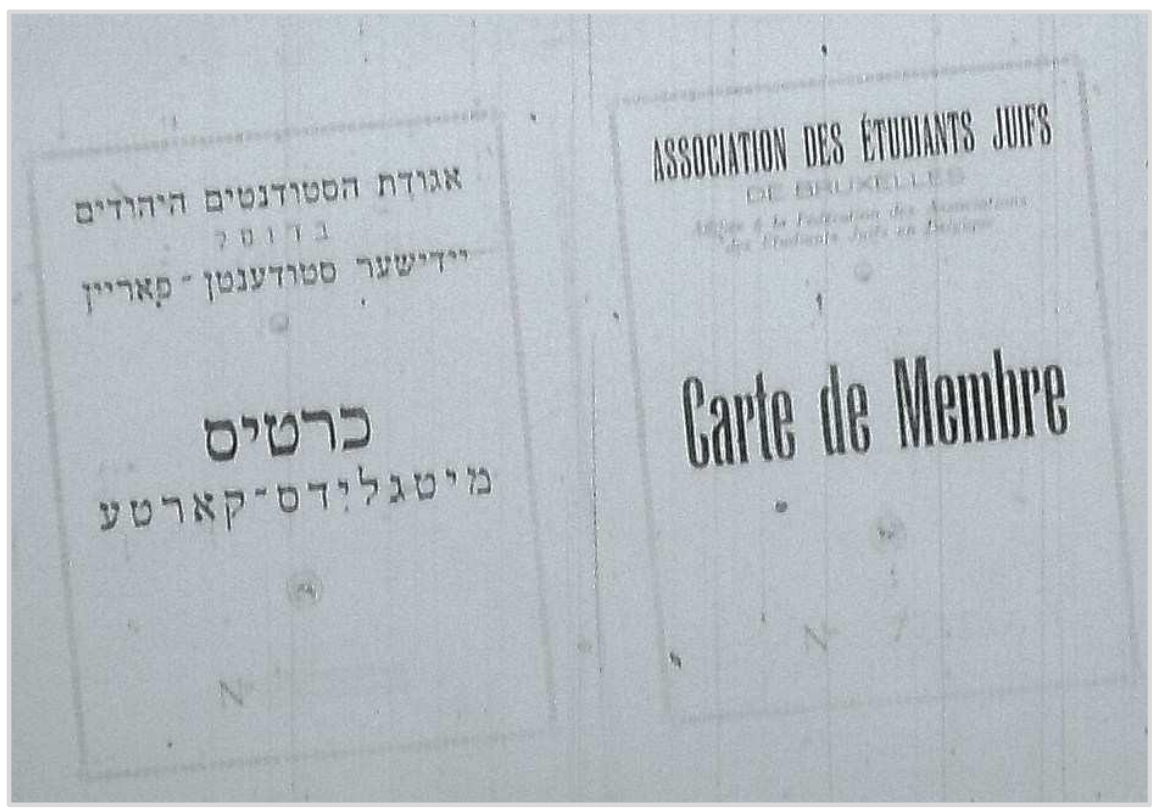

Carte de membre de l'Association des Étudiants juifs de Bruxelles (YIVO, New York, Collection David Trocki, dossier 34) [ill. 5]. 


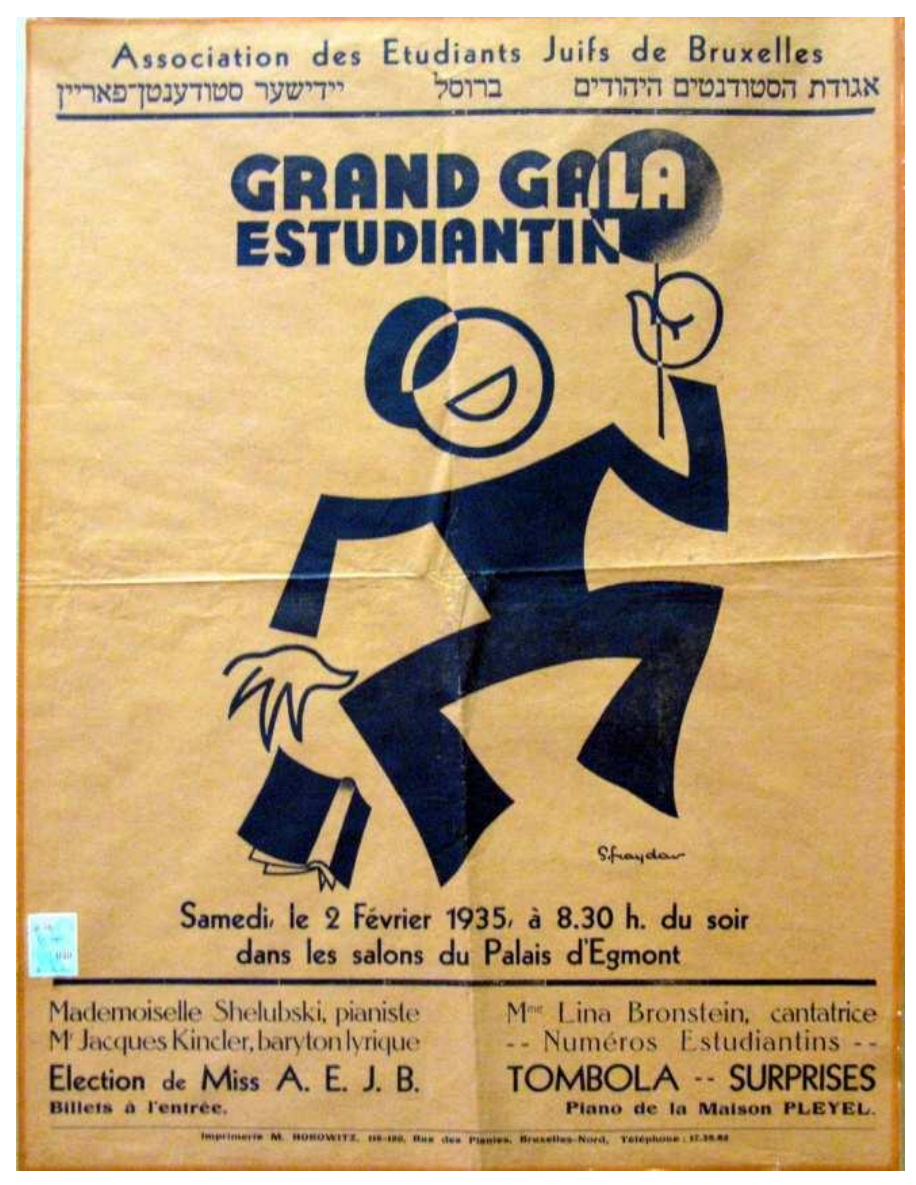

Annonce du Grand Gala estudiantin, 1935, Bruxelles (C) MJB) [ill. 6]. 


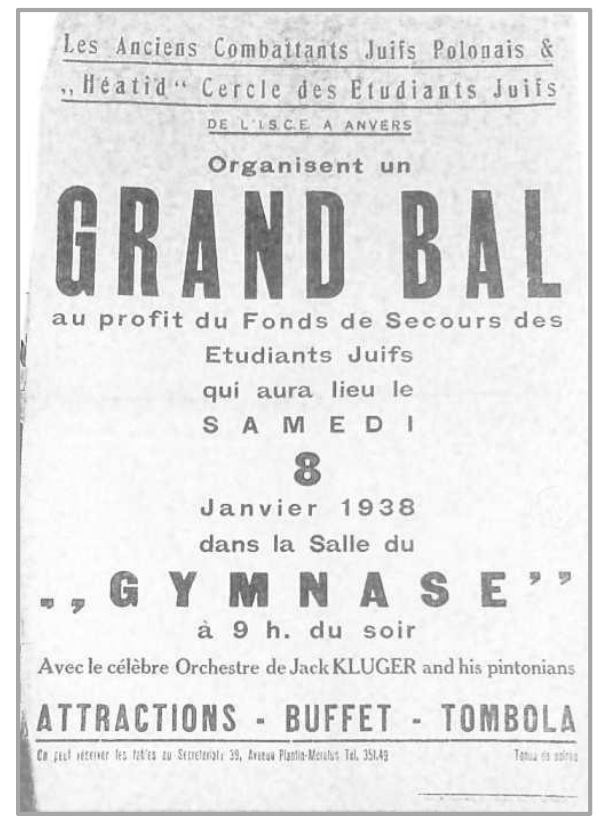

Annonce du bal organisé par les Anciens Combattants juifs polonais et Heatid,

l'Association des Étudiants juifs de l'ISCEA à Anvers, 8 janvier 1938 (en français et en yiddish) [ill. 7 et 8].

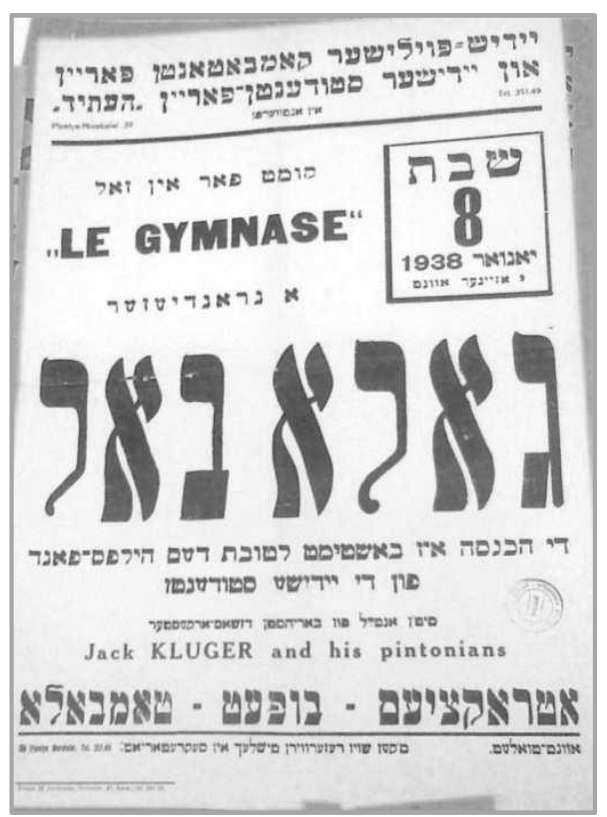

